P02.15.45

Acta Cryst. (2008). A64, C214

\section{Multitechnique solution of a new structural type $\mathrm{Bi3}^{\mathrm{Mn} 4}$ O11.22 ${ }^{\text {(NO3)0.93 }}$}

Olga Smirnova ${ }^{1}$, Azuma Masaki ${ }^{2}$, Nobuhiro Kumada ${ }^{1}$, Y Kusano ${ }^{3}$, Takahiro Takei ${ }^{1}$, Yoshinori Yonesaki ${ }^{1}$, Nobukazu Kinomura ${ }^{1}$ ${ }^{1}$ University of Yamanashi, Centre for Crystal Science and Technology, 7 Miyamae, Kofu, Yamanashi, 400-8511, Japan, ${ }^{2}$ Institute for Chemical Research, Kyoto University, ${ }^{3}$ Kurashiki University of Science and Arts, E-mail:smirnova@yamanashi.ac.jp

The black fine powder of the new compound was obtained by hydrothermal reaction; indexing of the electronic diffraction patterns provided the hexagonal cell. A combination of direct method of structure solution from powder (EXPO2004) and directspace method (FOX) using only synchrotron X-ray data allowed to locate heavy atoms in the structure, but provided very diffuse information about the light atoms. Further structure solution and Rietveld refinement (FullProf) using neutron data led to the non-centrosymmetric unit cell described in a space group P3 ( $a$ $=4.9679(3) \AA, c=13.161(1) \AA$ ) with one $\mathrm{NO}_{3}{ }^{-}$group per cell, significant distortion of $\mathrm{Mn}$ octahedra, and also random oxygen vacancies around $\mathrm{Bi}(1)$ atoms (Figure). $\mathrm{Bi}(1)$ has either five ligands or appears in a distorted octahedra; while $\mathrm{Bi}(2)$ and $\mathrm{Bi}(3)$ atoms have umbrellalike environment built of three oxygen ligands. The absence of the inversion centre was confirmed by the second harmonic generation measurement; the presence of $\mathrm{NO}_{3}^{-}$groups in the structure was confirmed by FT-IR measurements and TG Mass spectroscopy.

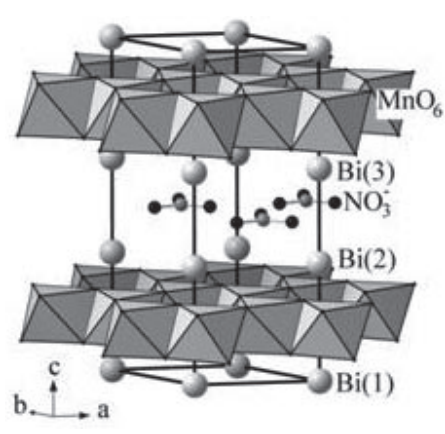

Keywords: electronic diffraction, X-ray diffraction, neutron diffraction

\section{P02.15.46}

Acta Cryst. (2008). A64, C214

The interaction between human rhinovirus $3 \mathrm{C}$ protease and stem loop $D$ studied by solution scattering

John Y H Chow ${ }^{1}$, Jolyon K Claridge ${ }^{2}$, Cy Jeffries ${ }^{1}$,

Stephen J Headley ${ }^{2}$, Giselle A Soares ${ }^{2}$, Martin Schwalbe ${ }^{2}$,

Steven M Pascal ${ }^{2}$, Jill Trewhella ${ }^{1}$

${ }^{1}$ University of Sydney, School of Molecular and Microbial Biosciences, Room 676, School of Molecular and Microbial Biosciences, Building G08, University of Sydney, New South Wales, 2006, Australia, ${ }^{2}$ Institute of Fundamental Sciences, Massey University, Palmerston North, New Zealand, E-mail:j.chow@mmb.usyd.edu.au

We present the results of recent small angle X-ray scattering and neutron contrast variation experiments that are part of a large effort to determine the structure of the $3 \mathrm{C}$ protease (3Cpro) and stem loop D (SLD) complex from human rhinovirus 14 (HRV-14). Human rhinoviruses are members of the picornaviridae family of small RNA viruses. Like all RNA viruses, picornaviridae achieve gene expression by inducing the host cell to produce a single polypeptide that is post-translationally cleaved to produce active viral proteins. In HRV-14 the role of cleaving viral proteins from the hostexpressed polypeptide is performed by 3 Cpro, which also acts as a crucial component of the HRV-14 ribonucleoprotein complex that initiates transcription of the viral genome during replication. The activity of this complex is dependent on the binding of 3Cpro to the cloverleaf structure known as SLD near the start of the 5' region of the genome. While the structure of the 3Cpro-SLD complex is unknown, individual structures of 3Cpro and SLD have been solved by X-ray diffraction and NMR. We have studied a 1:1 3Cpro-SLD complex using small-angle scattering and determined the ensemble average structure of the complex in solution. Comparisons between the structure of the complex and existing high resolution structures of the individual components will provide insights into conformational changes during the formation of the complex and assist in structurebased drug design.

Keywords: neutron contrast variation, SAXS, RNA-protein complexes

\section{P02.15.47}

Acta Cryst. (2008). A64, C214

\section{Inhibition of histidine kinase A in Bacillus subtilis: A neutron contrast variation study}

David A Jacques $^{1}$, David B Langley ${ }^{1}$, Andrew E Whitten ${ }^{2}$,

Cy M Jeffries $^{1}$, Jill Trewhella ${ }^{1}$

${ }^{1}$ University of Sydney, School of Molecular and Microbial Biosciences, Building G08 Butlin Avenue, University of Sydney, NSW, 2006, Australia, ${ }^{2}$ Australian Nuclear Science and Technology Organisation, Lucas Heights, NSW, Australia, E-mail:djac4793@mail.usyd.edu.au

We have used small-angle scattering with neutron contrast variation to investigate the interaction between Histidine Kinase A (KinA) and two of its inhibitors: Sda and KipI. In Bacillus subtilis, KinA is responsible for initiating a phosphorelay that culminates in the expression of genes controlling spore formation. The organism expresses $\mathrm{Sda}$ if it fails to replicate its chromosome, thereby preventing spore formation. Two Sda molecules then bind KinA and inhibit the autophosphorylation reaction. We have shown that Sda binds the dimerisation domain of KinA, which undergoes a conformational change resulting in a compaction of the KinA structure. While KipI is a much larger protein than Sda, and is expressed under different conditions, two KipI molecules also interact with the dimerisation domain of KinA resulting in the same compaction. Our molecular models for the inhibitor complexes (based on the crystal structures of homologous proteins) coupled with bioinformatic analyses of these systems show that the compaction is due to a collapse of the catalytic domains towards the dimerisation domains, which prevents the catalytic domains from appropriately accessing the target histidine. Our results also indicate that the dimerisation domain (a four-helix bundle) operates as a conduit for transmitting an inhibitory signal down the length of the molecule, an observation that has implications for the histidine kinases that are widely used in bacterial signal transduction.

Keywords: neutron contrast variation, small-angle scattering, signal transduction

\section{P02.15.48}

Acta Cryst. (2008). A64, C214-215

The KipI-KipA complex and histidine kinase regulation in Bacillus subtilis

David B Langley ${ }^{1}$, David A Jacques ${ }^{1}$, Andrew Whitten ${ }^{2}$, Jill Trewhella $^{1}$ 


\section{Poster Sessions}

${ }^{1}$ Sydney University, School of MMB, School of MMB, Building G08, Sydney University, Sydney, NSW, 2006, Australia, ${ }^{2}$ Australian Nuclear Science and Technology Organisation, Lucas Heights, Australia, E-mail : d.langley@mmb.usyd.edu.au

The decision to sporulate in Bacillus subtilis is primarily governed by the histidine kinase KinA instigating a phosphorelay resulting in the phosphorylation of the transcription factor Spo0A. The KipI protein is a regulator of this pathway, produced in response to low dietary nitrogen and high glucose conditions. KipI binds KinA, preventing the autophosphorylation required for the instigation of the phosphorelay. The KipA protein is expressed from the same operon as KipI and prevents its function as an inhibitor of KinA autokinase activity (an anti-anti-kinase). We have overexpressed and purified both proteins to homogeneity and demonstrated that they interact to form a complex. Biophysical investigation including small angle X-ray scattering and neutron contrast variation (using deuterated KipI) show that whilst the individual components are dimers at high concentration, when combined they form a 1:1 protein complex. The reconstructed shape of the KipI component closely resembles the structural envelope of a homologue ascribed as one subunit of an enzyme involved in urea metabolism - allophanate hydrolase. Although no structural model for KipA exists, at the amino acid level it resembles another allophante hydrolase subunit, raising the question of the evolution and function of these ubiquitous folds. Implications as to how anti-anti-kinase activity is achieved are discussed.

Keywords: small-angle scattering, neutron contrast variation, signal transduction

\section{P02.16.49}

Acta Cryst. (2008). A64, C215

\section{Synthesis, microstructure and catalytic property of} nanocrystalline $\mathrm{La}_{1-\mathrm{x}} \mathrm{Ce}_{\mathrm{X}} \mathrm{MnO}_{3}$

\section{Lam Dai Tran ${ }^{1}$, Hoang Vinh Tran ${ }^{1}$, Dang Hai Le ${ }^{2}$}

${ }^{1}$ Hanoi University of Technology, General and Inorganic Chemistry, C1-408, DHBK, 1, Dai Co Viet, Hanoi, Vietnam, 10, Vietnam, ${ }^{2}$ Hanoi Pedagogic University, 136, Xuan Thuy Road, Hanoi, Vietnam, E-mail : lamtd-fct@mail.hut.edu.vn

Manganites have attracted much attention of many researchers due to their fascinating physical and chemical properties, including catalytic one. In this study, Ce- doped nanocrystalline $\mathrm{La}_{1-\mathrm{x}} \mathrm{Ce}_{\mathrm{X}} \mathrm{MnO}_{3}$ $(x=0.05-0.2)$ powders have been prepared using sol - gel method at a relatively $\left(800^{\circ} \mathrm{C}\right)$. The crystal structure was examined by X-ray powder diffraction (XRD). The change in morphology, particle size and its surface area were also investigated by FE-SEM, TEM, SEM, BET measurements. Warren-Averbach and WilliamsonHall methods were used for microstructural analysis. The catalytic oxidation over hydrocarbon of nanopowders has been investigated.

Keywords: Ce-doped nanocrystalline $\mathrm{LaMnO}_{3}$, microstructure, catalytic property

\section{P02.16.50}

Acta Cryst. (2008). A64, C215

\section{Site preference of $\mathrm{Mn}$ in $\mathrm{Zn}_{2} \mathrm{SiO}_{4}$ phosphor by combined} Rietveld refinement

Yong-Il Kim, Lee Yun-Hee, Ryu Kwon-Sang, Lee Jeong-Soon

Korea Research Institute of Standards and Science, Korea Research
Institute of Standards and Science, P.O. Box 102, Yuseong, Daejeon, 305-600, Republic of Korea, Daejeon, Yuseong, 305-600, Korea (S), E-mail:yikim@kriss.re.kr

$\mathrm{Zn}_{2} \mathrm{SiO}_{4}: \mathrm{Mn}^{2+}$ as a green-color emitting phosphor which have an emission peak around $517 \mathrm{~nm}$ under UV excitation $(247 \mathrm{~nm})$ has been synthesized in solid state reaction. The combined Rietveld refinement was carried out to determine the site preference of $\mathrm{Mn}^{2+}$ ions in $\mathrm{Zn}_{2} \mathrm{SiO}_{4}$ phosphor. Of possible cation-disorder models, the best structural refinement result was obtained from a model that $\mathrm{Mn}^{2+}$ ions substitute for $\mathrm{Zn}^{2+}$ ions in $\mathrm{ZnO}_{4}$ tetrahedra. The model proposed by the combined Rietveld refinement was corroborated by the firstprinciple pseudopotential calculation. The converged weighted $R$-factor, $R_{\text {wp }}$, and the goodness-of-fit indicator, $S\left(=R_{\mathrm{wp}} / R_{\mathrm{e}}\right)$ were $9.17 \%$ and 2.40 , respectively. The occupancies of $\mathrm{Mn}^{2+}$ ions for two different $\mathrm{Zn}$ sites were 0.034(4) and 0.006(4), respectively. The refined model described a structure in space group $R 3$ (No.148) with $Z=18, a=b=13.9611(1) \AA, c=9.3294(1) \AA$ and $\gamma=120^{\circ}$.

Keywords: phosphors, Rietveld analysis, X-ray neutron powder diffraction

\section{P02.16.51}

Acta Cryst. (2008). A64, C215

\section{$A b$ initio structural characterization of $\mathrm{Bi}_{10} \mathrm{Mo}_{3} \mathrm{O}_{24}$ by TEM, X-ray and neutron powder diffraction}

Jorge Hernandez-Velasco ${ }^{1}$, A. Landa-Canovas ${ }^{1}$, E. Vila ${ }^{1}$, J. Galy ${ }^{2}$, A. Castro ${ }^{1}$

${ }^{1}$ Instituto de Ciencia de Materiales de Madrid, CSIC, Solidos Ionicos, Sor Juana Ines de la Cruz, 3. ICMM, CSIC Cantoblanco, Madrid, Madrid, 28049, Spain, ${ }^{2}$ Centre d'Etudes Structurales, CNRS Toulouse, France, E-mail:hernandez-velasco@hmi.de

$\mathrm{Bi}_{2} \mathrm{O}_{3}-\mathrm{MoO}_{3}$ system shows a full panoply of phases depending on $\mathrm{Bi} / \mathrm{Mo}$ ratio. They present great interest because of their catalytic and ionic conductivity properties. Among them, for $x \mathrm{Bi}_{2} \mathrm{O}_{3}: \mathrm{MoO}_{3}$ $1<x<2$ high temperature procedure for the chemical preparation yields mixtures, however single phases have been isolated by low $\mathrm{T}$ synthesis methods. In this sense we have characterized a new homologous series of oxides $\mathrm{Bi}_{2(n+2)} \mathrm{Mo}_{n} \mathrm{O}_{6(n+1)}$ with $\mathrm{n}=3,4,5$ and 6. For a deep understanding on the structure-property relationships there is a lack of crystallographic knowledge on these materials arising from the powder nature of the new obtained phases. Nevertheless, a multitechnique approach has been followed in order to solve their crystal structures. Electron diffraction patterns can be interpreted as a fluorite-type structure which gives rise to the basic lattice reflections, while weaker ones can be seen as satellites produced by a structural modulation. From HRTEM micrographs and using the metric relationship between the observed supercell and the basic fluorite one we infer cationic models for the new phases whose image simulations match well with the experimental images. We have used this cationic framework elucidation as starting point for the whole characterization of the structure by performing simultaneous Rietveld refinement of multipattern X-ray and neutron powder diffraction data, taking advantage of the neutron scattering length for $\mathrm{O}$ location. As example we show the simplest $\mathrm{Bi}_{10} \mathrm{Mo}_{3} \mathrm{O}_{24}$ structure with $5 \mathrm{Bi}, 2 \mathrm{Mo}$ and $13 \mathrm{O}$ different crystallographic positions in the asymmetric unit. It belongs to the monoclinic space group $C 2$ with $z=2$ and cell parameters: $a=23.7282(2) \AA, b=5.64906(6) \AA, c=8.68173(9) \AA, \beta=95.8668(7)$ $\circ$. It shows very good agreement between observed and calculated patterns.

Keywords: ab-initio structure determination, neutron and X-ray scattering, oxides ionic conductors 